\title{
Low-Level Detection of Poly(amidoamine) PAMAM Dendrimers Using Immunoimaging Scanning Probe Microscopy
}

\author{
Chevelle A. Cason, ${ }^{1}$ Thomas A. Fabré, ${ }^{1}$ Andrew Buhrlage, ${ }^{1}$ \\ Kristi L. Haik, ${ }^{2}$ and Heather A. Bullen ${ }^{1}$ \\ ${ }^{1}$ Department of Chemistry, Northern Kentucky University, Highland Heights, KY 41099, USA \\ ${ }^{2}$ Department of Biological Sciences, Northern Kentucky University, Highland Heights, KY 41099, USA
}

Correspondence should be addressed to Heather A. Bullen, bullenh1@nku.edu

Received 29 September 2011; Accepted 3 November 2011

Academic Editor: Charles L. Wilkins

Copyright (C) 2012 Chevelle A. Cason et al. This is an open access article distributed under the Creative Commons Attribution License, which permits unrestricted use, distribution, and reproduction in any medium, provided the original work is properly cited.

\begin{abstract}
Immunoimaging scanning probe microscopy was utilized for the low-level detection and quantification of biotinylated G4 poly(amidoamine) PAMAM dendrimers. Results were compared to those of high-performance liquid chromatography (HPLC) and found to provide a vastly improved analytical method for the low-level detection of dendrimers, improving the limit of detection by a factor of 1000 (LOD $=2.5 \times 10^{-13}$ moles). The biorecognition method is reproducible and shows high specificity and good accuracy. In addition, the capture assay platform shows a promising approach to patterning dendrimers for nanotechnology applications.
\end{abstract}

\section{Introduction}

Dendrimers are at the forefront of research in nanoscience due to the many interesting properties of these macromolecular systems including their precise architecture, highly reproducible shape, high uniformity and purity, low immunogenicity and toxicity, high loading capacity, and high shear resistance [1-5]. They have shown a great deal of versatility with applications in numerous areas such as drug delivery $[6,7]$, gene therapy $[8,9]$, chemotherapy [10], electrochemistry $[11,12]$, metal recovery [13], catalysis $[14,15]$, and sensors [16-18]. Development of new low-level detection and quantification methods is needed with the utilization of these nanomaterials. Currently, high-performance liquid chromatography (HPLC) is the predominate approach reported for dendrimer quantification $[19,20]$. However, the primary focus of HPLC, along with capillary electrophoresis, has been to evaluate dendrimer purity and degree of conjugation [21-27]. Little has been reported within the literature with regard to the advancement of new quantification methods for dendrimers.

This work introduces a biorecognition readout technique that has the potential to provide low-level detection of dendrimers. Biotinylated poly(amidoamine) PAMAM dendrimers were chosen as a model target. PAMAM dendrimers, which are highly water soluble, represent the most widely studied class of dendrimers. Functionalization of PAMAM dendrimer surfaces has proven useful in their utilization for various applications including drug delivery and chemical sensing $[5,6,16]$. Biotin-labeled dendrimers have been utilized in tumor [28] and antibody [29] targeting studies and biosensor design [30]. Biotinylated PAMAM dendrimers may also have the potential for delivering therapeutic drugs to the brain $[31,32]$.

We report here a readout method using an immunoassay platform and scanning probe microscopy (SPM) for lowlevel quantification of biotinylated G4 PAMAM dendrimers. The assay takes advantage of the documented specificity of biotin-avidin. Results are correlated with HPLC analysis. In addition, we briefly highlight the potential of this capture assay platform to selectively pattern PAMAM dendrimers onto a surface. Patterning of nanoparticles is relevant to a wide variety of applications in the fields of sensing, drug delivery, or development of nanodevices [33-35]. Dendritic architectures show promise in designing and developing sensor platforms with high sensitivity and stability [16]. 


\section{Experimental}

2.1. Reagents. Poly(amidoamine) PAMAM dendrimers [core: ethylene diamine] $(\mathrm{G}=4)$ dendri-PAMAM- $\left(\mathrm{NH}_{2}\right)_{32}$ were obtained from Dendritic Nanotechnologies, Inc. (Mt. Pleasant, MI). Biotinylated PAMAM dendrimers were prepared using sulfo-NHS-LC-biotin (Pierce EZ-Link Kit) as described previously [36]. Briefly, a 3:1 molar ratio of biotin/PAMAM dendrimers in $0.1 \mathrm{M}$ phosphate buffer saline (PBS) was allowed to react for $2 \mathrm{~h}$ on an orbital shaker. Excess, unreacted biotin was then removed using Microcon filters (Millipore. Bedford, MA, USA). The biotinylation of dendrimers was evaluated using NMR spectroscopy. Biotinylated dendrimers were resuspended $(1.0 \mathrm{mg} / \mathrm{mL})$ in 1.0 M PBS until used. Octadecanethiol (ODT), 3,3'dithio-bis(propionic acid N-hydroxysuccinimide ester) (DSP), bovine serum albumin (BSA), Triton X-100, and avidin $>98 \%$ were obtained from Sigma (Sigma-Aldrich, St. Louis, MO). Avidin conjugated to Alexa Fluor 488 was purchased from Invitrogen (Invitrogen, Carlsbad, CA). Poly(dimethyl siloxane) (PDMS) was obtained from Dow Corning (Midland, MI). All organic solvents used were analytical, HPLC grade, from Sigma (Sigma-Aldrich, St. Louis, MO). DI water was obtained using a Milli-Q plus water purification system (Millipore, Bedford, MA). PBS and Borate buffers were prepared from Pierce buffer packs (Pierce Protein Research Products, Rockford, IL).

2.2. Capture Substrate Preparation. A modified approach was used for preparation of the capture substrate [37-39] utilizing template-stripped gold (TSG) for SPM imaging, as shown in Figure 1. TSG was prepared by evaporating gold onto p-type silicon wafers (University Wafer) with a resistive evaporator and affixing $1 \times 1 \mathrm{~cm}$ glass pieces (ultrasonically cleaned $30 \mathrm{~min}$ each in diluted Contrad 70, DI water, and methanol) using two-part epoxy (Epoxy Technology) followed by curing at $150^{\circ} \mathrm{C}$ for $2 \mathrm{~h}$. The glass pieces were gently detached from the silicon wafer revealing a smooth gold surface atop the glass chip.

The TSG substrates were exposed for $\sim 30 \mathrm{~s}$ to an ODT soaked PDMS stamp (with a $3 \mathrm{~mm}$ diameter hole cut in the center), rinsed with ethanol, and dried under highpurity nitrogen. The substrates were then placed in a $0.1 \mathrm{mM}$ solution of DSP in ethanol overnight. The capture platform was then rinsed with ethanol and dried under $\mathrm{N}_{2}$. This formed the DSP-based adlayer in the areas on the substrate not covered by ODT. The hydrophobic ODT localized reagents in a confined sample area ( $3 \mathrm{~mm}$ ODT spot size) for the capture assay platform.

To form the capture avidin surface, a $20 \mu \mathrm{L}$ aliquot of avidin solution $(500 \mu \mathrm{g} / \mathrm{mL}$ diluted in $50 \mathrm{mM}$ borate buffer) was placed on top of the sample area and allowed to incubate for $6 \mathrm{~h}$ at room temperature in a humidity chamber. Substrates were then rinsed with $5 \mathrm{~mL}$ of $10 \mathrm{mM}$ PBS (with $0.1 \%$ Triton $\mathrm{X}-100)$, and the surface area was incubated with a $20 \mu \mathrm{L}$ solution of blocking buffer (1\% (w/v) BSA in $20 \mathrm{mM}$ borate buffer with $0.1 \%$ Triton $\mathrm{X}$ ) for $5 \mathrm{~h}$, followed by rinsing with $5 \mathrm{~mL}$ of $10 \mathrm{mM}$ PBS. The capture substrate was then exposed to $20 \mu \mathrm{L}$ aliquots of various concentrations

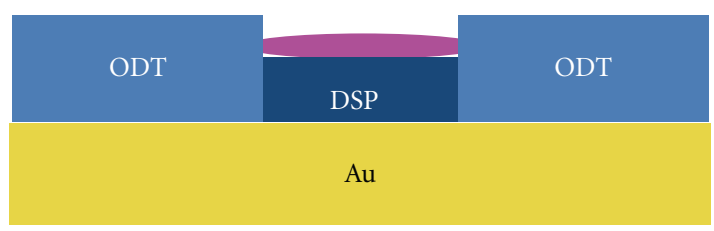

(a)

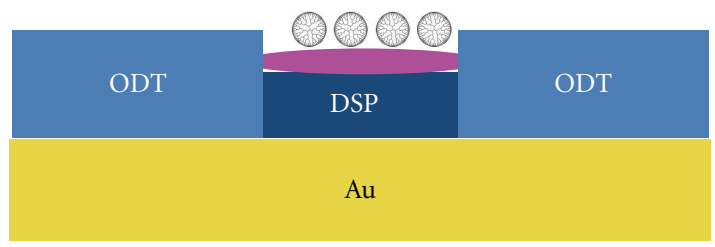

(b)

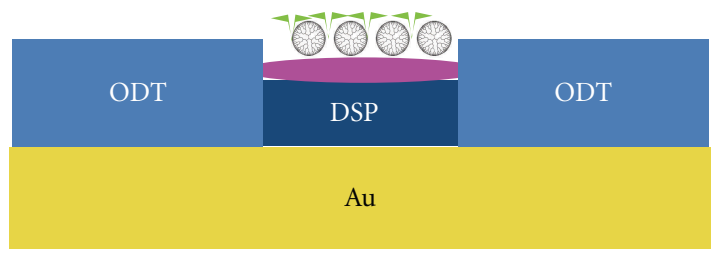

(c)

DSP: Dithiobis (succinimidyl propionate) ODT: Octadecanethiol

$$
\begin{aligned}
& \text { : Avidin } \\
& \text { : Biotinylated PAMAM } \\
& \text { : Fluorescently tagged avidin }
\end{aligned}
$$

FIgURE 1: Schematic of capture assay platform: (a) immobilize avidin onto Au substrate with controlled surface area using DSP and (b) expose substrate to biotinylated PAMAM dendrimers. (c) The captured biotinylated PAMAM dendrimers can be fluorescently labeled for additional verification of immobilization and patterning of dendrimers.

of biotinylated G4 PAMAM dendrimers diluted in $10 \mathrm{mM}$ PBS for $8 \mathrm{~h}$ at room temperature in a humidity chamber. Substrates were then rinsed with $5 \mathrm{~mL}$ of $10 \mathrm{mM}$ borate buffer before drying with nitrogen. A blank sample consisted of all stages except the avidin capture surface.

To demonstrate patterning of biotinylated G4 PAMAM dendrimers, a PDMS stamp with a positive structure of $10 \mu \mathrm{m}$ wide stripes separated by $5 \mu \mathrm{m}$ was utilized. Preparation of the patterned substrate consisted of all stages, with the following modifications: (1) a sample volume of $200 \mu \mathrm{L}$ was used instead of $20 \mu \mathrm{L}$ and (2) after incubation with biotinylated G4 PAMAM dendrimers, the substrate was rinsed with $10 \mathrm{mM}$ PBS and the dendrimers were fluorescently tagged by incubating the substrate with $200 \mu \mathrm{L}$ of $250 \mathrm{ug} / \mathrm{mL}$ of Alexa Fluor conjugated avidin. Following this step the substrates were rinsed with $5 \mathrm{~mL}$ of $10 \mathrm{mM}$ borate buffer and dried with nitrogen.

\subsection{Scanning Probe Microscopy. A Dimension 3100 Digital} Instruments SPM was utilized in tapping mode equipped with $125 \mu \mathrm{m}$ n-doped silicon cantilevers with resonance frequencies between 110 and $220 \mathrm{kHz}$ and typical scan rates 


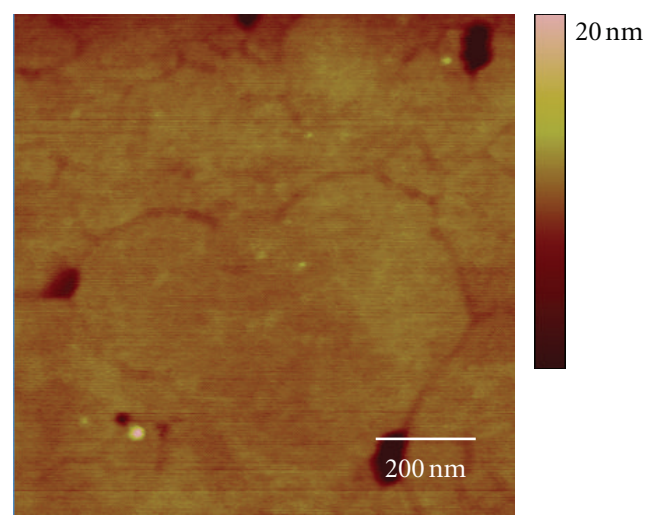

(a)

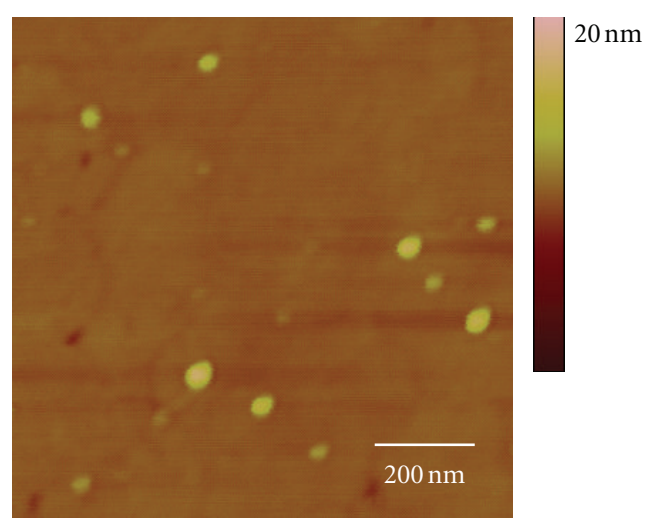

(c)

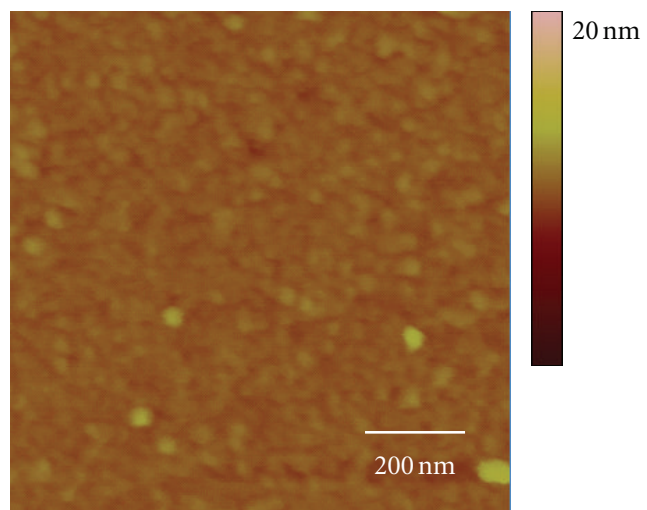

(b)

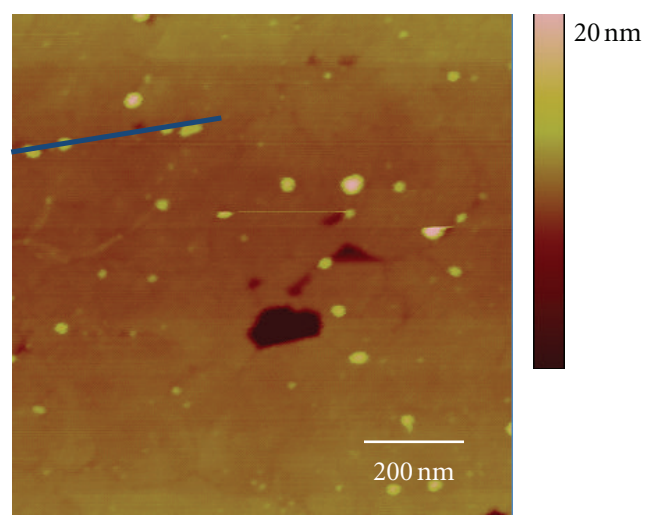

(d)

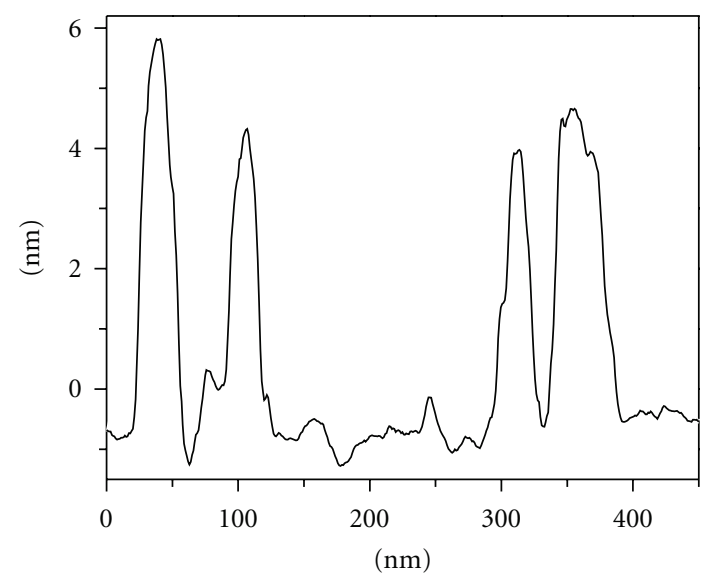

(e)

FIGURE 2: Representative SPM images of capture surface $(1 \times 1 \mu \mathrm{m})$ of different concentrations of bound biotinylated G4 PAMAM dendrimers to avidin capture substrate: (a) blank and (b) $7.0 \times 10^{-3} \mu \mathrm{mol} / \mathrm{L}$, (c) $7.0 \times 10^{-2} \mu \mathrm{mol} / \mathrm{L}$, and (d) $3.28 \times 10^{-1} \mu \mathrm{mol} / \mathrm{L}$; (e) a cross-sectional analysis of captured biotinylated G4 PAMAM dendrimers in (d).

of $0.75-1 \mathrm{~Hz}$ (NSC 14, MikroMasch). Typical scan rates were $1 \mathrm{~Hz}$, and all images were acquired in air. For quantification, multiple areas of the capture surface were analyzed and the dendrimers in each $1 \times 1 \mu \mathrm{m}$ image were enumerated manually.

2.4. High-Performance Liquid Chromatography. HPLC analysis of G4 biotinylated PAMAM dendrimers was conducted using parameters reported previously [36]. Briefly, a reversed phase HPLC system consisting of a Waters Breeze HPLC (Waters Corporation, Milford, MA, USA), equipped with a 717plus autosampler, 2487 dual $\lambda$ UV detector, $5 \mu \mathrm{m}$ Symmetry300 C18 column $(4.6 \mathrm{~mm} \times 150 \mathrm{~mm})$, and a Waters Sentry Symmetry C18 guard column was used. The mobile phase consisted of a linear gradient beginning with $90 \%$ water and $10 \% \mathrm{ACN}$ (each with $0.14 \% \mathrm{TFA}$ ) at a flow 
rate of $1 \mathrm{~mL} / \mathrm{min}$ reaching $68 \%$ water and $32 \% \mathrm{ACN}$ over 10 minutes. The injection volume was $100 \mu \mathrm{L}$, and detection of eluted samples was performed at $214 \mathrm{~nm}$.

2.5. Fluorescent Microscopy. A Nikon E600 fluorescent microscope with a Mercury-100 W light source was utilized to take images at $100 \mathrm{x}$ total magnification.

\section{Results and Discussion}

The principle goal of this work was the development and application of capture platform for quantification of PAMAM dendrimers. A model assay was evaluated using biotinylated G4 PAMAM dendrimers and avidin. Avidin was immobilized onto a patterned thiolate layer, DSP, via succinimidyl ester chemistry (Figure 1). In the presence of biotinylated G4 PAMAM dendrimers, the immobilized avidin layer extracts the dendrimers. SPM analysis of the capture platform revealed the presence of nanometrically sized objects, $\sim 4.5 \mathrm{~nm}$ in dimension, according to crosssectional height profiles. These are consistent in size and shape of G4 biotinylated PAMAM dendrimers, taking into account tip convolution effects (Figure 2). At this scale, the tip convolution effects distorted the resolution in the lateral dimensions, as the dimensions of the particles are smaller than the radius of curvature of the AFM probe used (radius = $20 \mathrm{~nm})[40,41]$. The dendrimers largely appear to be captured individually on the platform surface. In some cases dendrimers were found grouped together (2-3 dendrimer clusters) but could be individually distinguished using crosssectional analysis. SPM analysis reveals that the dendrimers do not have a tendency to cluster together in domains, indicating that sedimentation is not a factor during the incubation. Analysis of control (blank) substrates, devoid of the avidin-specific capture surface, shows limited nonspecific binding $\left(0.67 \pm 0.45\right.$ dendrimers $\left./ \mu \mathrm{m}^{2}\right)$ and that blocking steps are effective.

The patterning of DSP provides a reproducible surface area for quantification. Figure 2 shows that the number of captured G4 biotinylated PAMAM dendrimers tracks with the increase in dendrimer solution concentration. The degree of bound dendrimers was similar for various different areas of the capture surface. A calibration plot of the amount of captured G4 biotinylated PAMAM dendrimers (determined by SPM analysis) as a function of solution concentration is shown in Figure 3(a). The number of captured dendrimers correlates with G4 biotinylated PAMAM dendrimer concentrations, showing a linear dynamic range under the concentrations range evaluated $\left(7.0 \times 10^{-3}-3.28 \times\right.$ $\left.10^{-1} \mu \mathrm{mol} / \mathrm{L}\right)$. This readout method has the potential for low limits of detection. The limit of detection can be estimated as the signal three times above the measurement noise, where the noise is the sample blank response. This corresponds to a limit of detection of $\sim 2.5 \times 10^{-13}$ moles. Results were correlated with a standard HPLC analysis approach for quantification of dendrimers, as shown in Figure 3(b). HPLC quantification of dendrimers shows a linear dynamic range under the concentrations evaluated

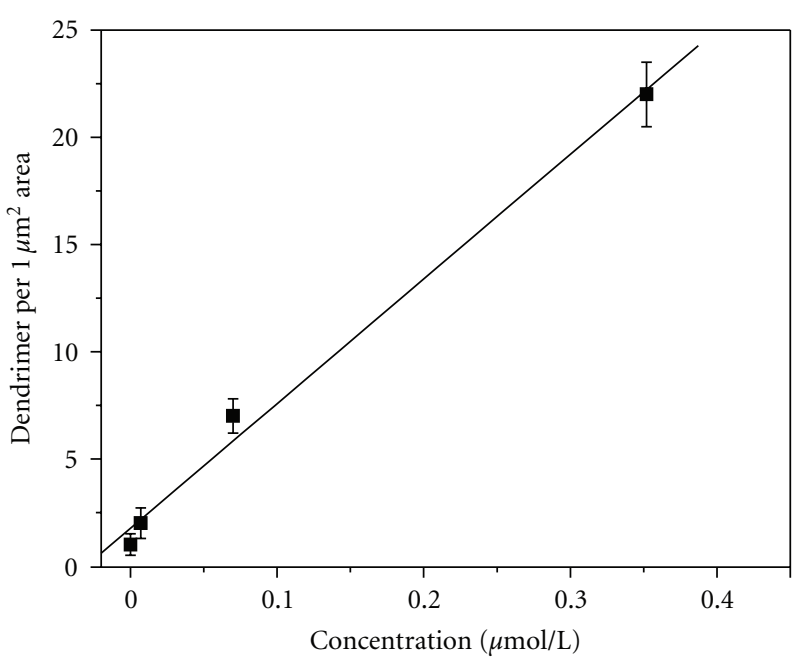

(a)

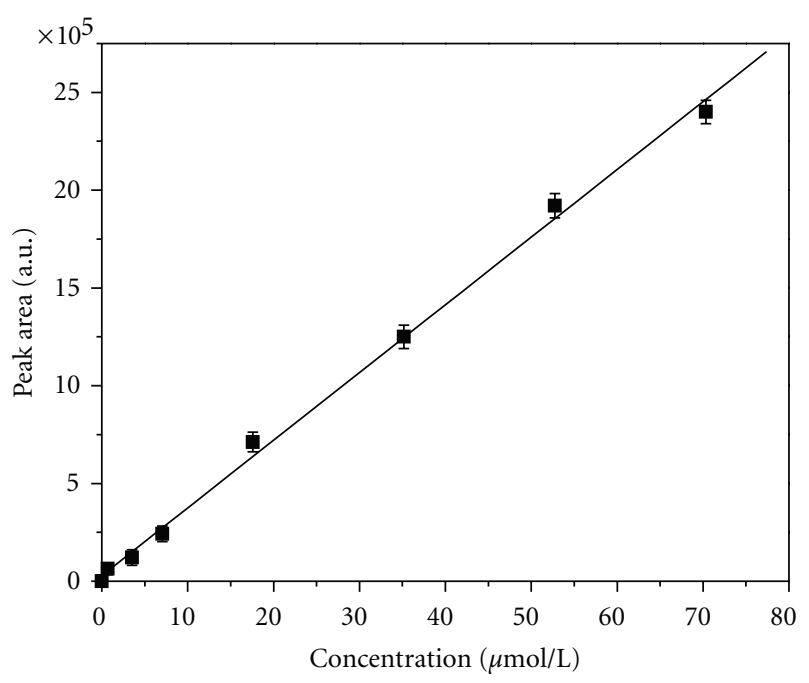

(b)

FIgure 3: Calibration curves for (a) immunoimaging SPM assay and (b) HPLC analysis of G4 biotinylated PAMAM dendrimers. The SPM analysis represents the average number of dendrimers bound within a $1 \times 1 \mu \mathrm{m}$ area for different concentrations of dendrimers. Data acquired from 10 capture substrates, with 10 images per sample $\left(y=58.1 x+1.76, n=20, r^{2}=0.9964\right)$. The HPLC analysis represents an average of 10 samples for each concentration evaluated $\left(y=34620.85 x+28845.984, n=10, r^{2}=0.9986\right)$.

$\left(7.0 \times 10^{-1}-70.35 \mu \mathrm{mol} / \mathrm{L}\right)$ with a corresponding limit of detection of $1.8 \times 10^{-10}$ moles. The capture assay platform improves the limit of detection by a factor of 1000. It should also be noted that the concentrations investigated by the capture assay were not detectable by HPLC analysis.

The capture assay platform has shown the potential for low-level detection and quantification of G4 biotinylated PAMAM dendrimers. Further improvements in the performance of this immunosorbent assay approach such as the reduction of incubation times may be possible by utilizing a rotating capture substrate to increase the flux of analyte to the capture surface [38]. In addition, the limit of detection may be further improved by reducing the area dimension 


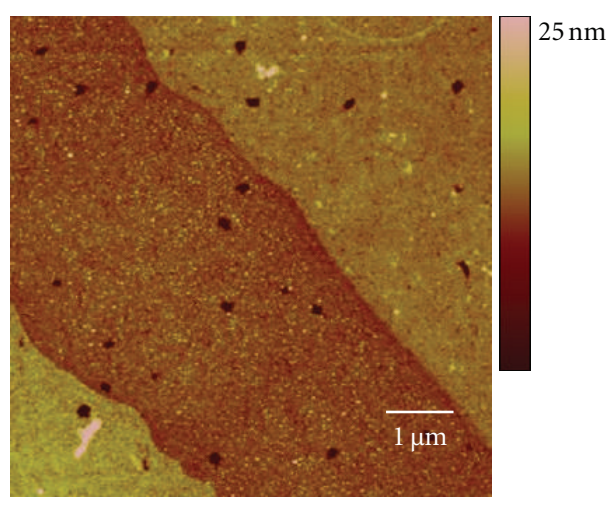

(a)

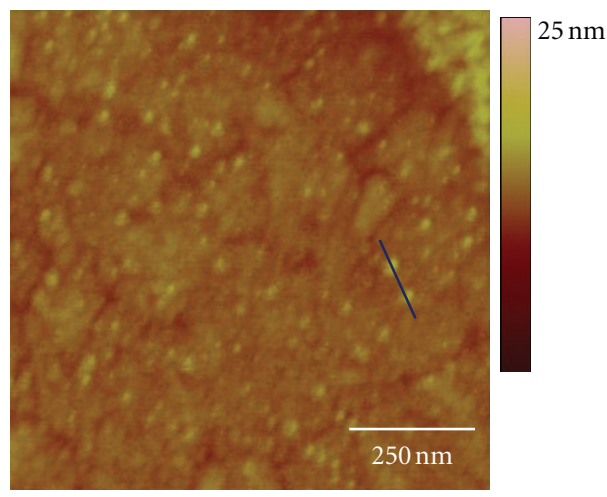

(c)

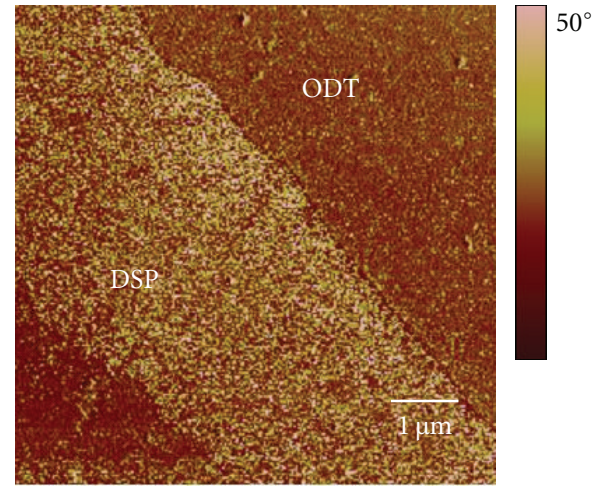

(b)

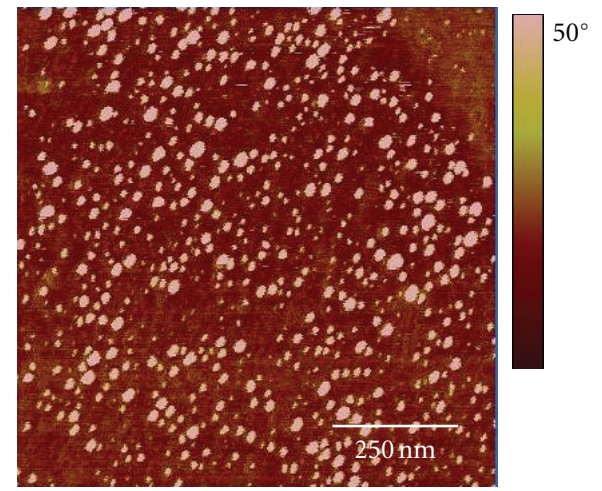

(d)

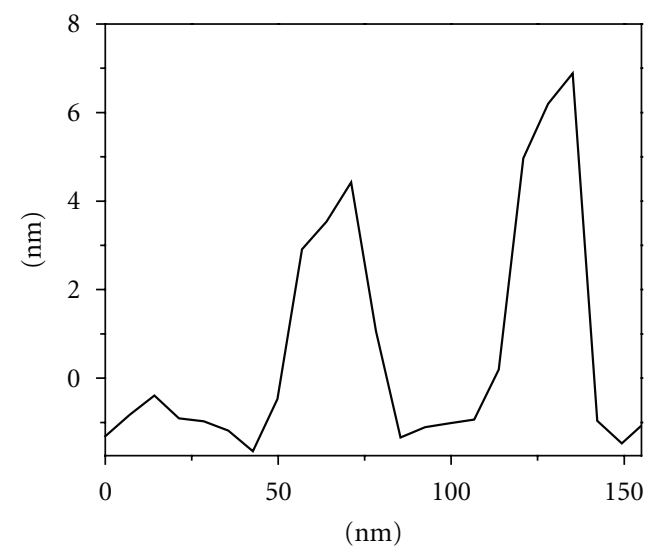

(e)

FIGURE 4: SPM image of capture surface patterning: (a) height and (b) phase $(8 \times 8 \mu \mathrm{m})$, zoomed in analysis; (c) height and (b) phase of capture stripe (avidin immobilized on DSP) containing bound G4 biotinylated PAMAM dendrimers. Cross-section analysis of bound G4 biotinylated PAMAM dendrimers in (d) is shown in (e). The capture platform was exposed to $200 \mu \mathrm{L}$ of $5.0 \mu \mathrm{mol} / \mathrm{L}$ concentration of G4 biotinylated PAMAM dendrimer solution.

of the DSP capture platform. The range of dendrimers that can be quantified using this approach is potentially limited to the analyte capture surface and labeling strategy of the dendrimers. This quantification method can be applied to any type of immunosorbant assay approach (e.g., antibody antigen binding). In addition, the capture platform design could be utilized for multidetection capability. It is important to note, however, that quantification of unfunctionalized dendrimers would require a labeling strategy for this capture assay approach to be used.
The capture assay platform also has the ability to selectively pattern PAMAM dendrimers onto a surface, as shown in Figure 4. In this study a PDMS stamp with a positive structure of $10 \mu \mathrm{m}$ wide stripes separated by $5 \mu \mathrm{m}$ stripes was used to pattern G4 biotinylated PAMAM dendrimers using the assay approach. Figures 4(a) and 4(b) show the height and phase analysis of the patterned surface. The bright stripes in the height analysis (Figure 4(a)) correspond to the ODT layer $(10 \mu \mathrm{m}$ wide), and the darker stripe corresponds to the DSP layer ( $5 \mu \mathrm{m}$ wide). The height difference $(\sim 2.5 \mathrm{~nm})$ 


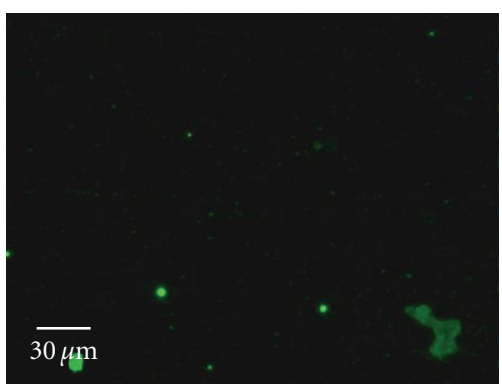

(a)

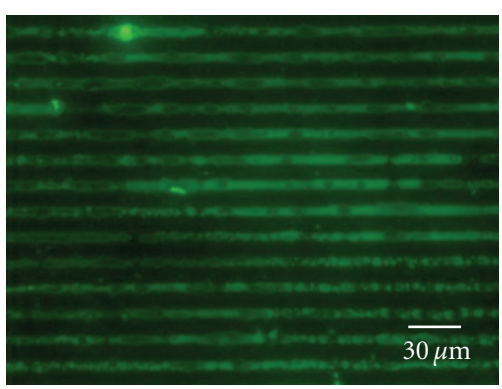

(b)

FIGURE 5: Fluorescence image of capture surface assay utilizing Alexa Fluor conjugated avidin: (a) blank and (b) G4 biotinylated PAMAM dendrimers bound to avidin capture substrate.

is consistent with the differing dimensions of ODT and DSP monolayers. It can be seen from the phase analysis that the captured dendrimers are more easily identifiable in the DSP stripe (Figure 4(b)). Figures 4(c) and 4(d) present higher-resolution height and phase analysis of the patterned G4 biotinylated PAMAM dendrimers. In these SPM images the individual dendrimers bound to the DSP stripe can be ascertained. The dendrimers are individually distributed within the DSP stripe. The dendrimers do not clump together and multilayers of dendrimers are not evident. Furthermore, the ODT layers do not show any evidence of nonspecific adsorption. Cross-sectional height profiles $(\sim 4.5 \mathrm{~nm})$ are consistent with $\mathrm{G} 4$ dendrimers. The slight variabilities in the height may be due to variations in how the biotin on the dendrimer surface is bound to the avidin capture platform. Further validation of the patterning of the G4 biotinylated PAMAM dendrimers was conducted by tagging the bound dendrimers with Alexa Fluor conjugated avidin, as shown in Figure 5. Fluorescence imaging verifies that the dendrimers are patterned on the avidin capture surface over large surface areas. Analysis of the blank confirms that nonspecific adsorption of G4 biotinylated PAMAM dendrimers is minimal. The bound patterned dendrimers have been found to be stable on the capture platform for $>1$ year under ambient storage conditions. Although, methods such as direct microcontact printing [42] dip-pen lithography [43], and electron-beam patterning [44] of dendrimers have been reported previously, they do not provide the selective patterning of this approach. The dimensions and profiles of the patterned dendrimers can be modified using different PDMS platforms.

\section{Conclusions}

This paper has demonstrated the potential value of immunoimaging SPM to detect and quantify G4 biotinylated PAMAM dendrimers. Results were presented that showed that this new approach using an immunoassay platform provides a 1000-fold improvement in the limit of detection of dendrimers compared to current quantification methods using HPLC. The range of dendrimers that could be evaluated with this approach is potentially limited to the availability of analyte capture and labeling strategies. Findings have also shown the potential of this platform to pattern dendrimers for sensor and nanodevice applications.

\section{Acknowledgments}

This project has been funded by the Merck Institute for Science Education, Kentucky NSF EPSCoR, Northern Kentucky University, Center for Integrated Natural Science and Mathematics, and the NKU Research Foundation. This work was also supported by a grant from the Kentucky Biomedical Research Infrastructure Network (P20 RR016481-08).

\section{References}

[1] G. M. Dykes, "Dendrimers: a review of their appeal and applications," Journal of Chemical Technology and Biotechnology, vol. 76, no. 9, pp. 903-918, 2001.

[2] D. A. Tomalia, "Birth of a new macromolecular architecture: dendrimers as quantized building blocks for nanoscale synthetic polymer chemistry," Progress in Polymer Science, vol. 30, no. 3-4, pp. 294-324, 2005.

[3] D. A. Tomalia, "The emergence of a new macromolecular architecture: the dendritic state," in Physical Properties of Polymers Handbook, J. E. Mark, Ed., pp. 671-692, Springer, New York, NY, USA, 2007.

[4] D. A. Tomalia, S. A. Henderson, and M. S. Diallo, "Dendrimers-an enabling synthetic science to controlled organic nanostructures," in Handbook of Nanoscience, Engineering and Technology, W. A. I. Goddard, D. W. Brenner, S. E. Lyshevski, and G. J. Irafrate, Eds., pp. 24.1-24.47, CRC Press, Taylor and Francis, Boca Raton, Fla, USA, 2007.

[5] D. Astruc, E. Boisselier, and C. Ornelas, "Dendrimers designed for functions: from physical, photophysical, and supramolecular properties to applications in sensing, catalysis, molecular electronics, photonics, and nanomedicine," Chemical Reviews, vol. 110, no. 4, pp. 1857-1959, 2010.

[6] V. Gajbhiye, V. K. Palanirajan, R. K. Tekade, and N. K. Jain, "Dendrimers as therapeutic agents: a systematic review," Journal of Pharmacy and Pharmacology, vol. 61, no. 8, pp. 9891003, 2009.

[7] W. Wijagkanalan, S. Kawakami, and M. Hashida, "Designing dendrimers for drug delivery and imaging: pharmacokinetic considerations," Pharmaceutical Research, vol. 28, no. 7, pp. 1500-1519, 2011.

[8] K. C. Petkar, S. S. Chavhan, S. Agatonovik-Kustrin, and K. K. Sawant, "Nanostructured materials in drug and gene delivery: a review of the state of the art," Critical Reviews in Therapeutic Drug Carrier Systems, vol. 28, no. 2, pp. 101-164, 2011.

[9] M. A. Mintzer and M. W. Grinstaff, "Biomedical applications of dendrimers: a tutorial," Chemical Society Reviews, vol. 40, no. 1, pp. 173-190, 2011. 
[10] Y. Cheng, L. Zhao, Y. Li, and T. Xu, "Design of biocompatible dendrimers for cancer diagnosis and therapy: current status and future perspectives," Chemical Society Reviews, vol. 40, no. 5, pp. 2673-2703, 2011.

[11] E. Bustos, J. Manríquez, G. Orozco, and L. A. Godínez, "Preparation, characterization, and electrocatalytic activity of surface anchored, Prussian Blue containing starburst PAMAM dendrimers on gold electrodes," Langmuir, vol. 21, no. 7, pp. 3013-3021, 2005.

[12] N. Zhu, Y. Gu, Z. Chang, P. He, and Y. Fang, "PAMAM dendrimers-based DNA biosensors for electrochemical detection of DNA hybridization," Electroanalysis, vol. 18, no. 21, pp. 2107-2114, 2006.

[13] M. S. Diallo, S. Christie, P. Swaminathan, J. H. Johnson Jr., and W. A. Goddard III, "Dendrimer enhanced ultrafiltration-1. Recovery of $\mathrm{Cu}$ (II) from aqueous solutions using PAMAM dendrimers with ethylene diamine core and terminal $\mathrm{NH} 2$ groups," Environmental Science and Technology, vol. 39, no. 5, pp. 1366-1377, 2005.

[14] L. J. Twyman, A. S. H. King, and I. K. Martin, "Catalysis inside dendrimers," Chemical Society Reviews, vol. 31, no. 2, pp. 6982, 2002.

[15] J. N. H. Reek, D. De Groot, G. E. Oosterom, P. C. J. Kamer, and P. W. N. M. Van Leeuwen, "Core and periphery functionalized dendrimers for transition metal catalysis; a covalent and a non-covalent approach," Reviews in Molecular Biotechnology, vol. 90, no. 3-4, pp. 159-181, 2002.

[16] J. Satija, V. V. R. Sai, and S. Mukherji, "Dendrimers in biosensors: concept and applications," Journal of Materials Chemistry, vol. 21, pp. 14367-14386, 2011.

[17] J. Losada, M. Zamora, P. García Armada, I. Cuadrado, B. Alonso, and C. M. Casado, "Bienzyme sensors based on novel polymethylferrocenyl dendrimers," Analytical and Bioanalytical Chemistry, vol. 385, no. 7, pp. 1209-1217, 2006.

[18] H. Cavaye, P. E. Shaw, A. R.G. Smith et al., "Solid state dendrimer sensors: effect of dendrimer dimensionality on detection and sequestration of 2,4-dinitrotoluene," Journal of Physical Chemistry C, vol. 115, no. 37, pp. 18366-18371, 2011.

[19] J. Giri, M. S. Diallo, W. A. Goddard, N. F. Dalleska, X. Fang, and Y. Tang, "Partitioning of poly(amidoamine) dendrimers between n-octanol and water," Environmental Science and Technology, vol. 43, no. 13, pp. 5123-5129, 2009.

[20] D. G. Mullen, E. L. Borgmeier, A. M. Desai et al., "Isolation and characterization of dendrimers with precise numbers of functional groups," Chemistry, vol. 16, no. 35, pp. 1067510678, 2010.

[21] S. Mutalik, A. K. Hewavitharana, P. N. Shaw, Y. G. Anissimov, M. S. Roberts, and H. S. Parekh, "Development and validation of a reversed-phase high-performance liquid chromatographic method for quantification of peptide dendrimers in human skin permeation experiments," Journal of Chromatography B, vol. 877, no. 29, pp. 3556-3562, 2009.

[22] S. Lalwani, V. J. Venditto, A. Chouai, G. E. Rivera, S. Shaunak, and E. E. Simanek, "Electrophoretic behavior of anionic triazine and pamam dendrimers: methods for improving resolution and assessing purity using capillary electrophoresis," Macromolecules, vol. 42, no. 8, pp. 3152-3161, 2009.

[23] M. T. Islam, I. J. Majoros, and J. R. Baker Jr., "HPLC analysis of PAMAM dendrimer based multifunctional devices," Journal of Chromatography B, vol. 822, no. 1-2, pp. 21-26, 2005.

[24] M. T. Islam, X. Shi, L. Balogh, and J. R. Baker, "HPLC separation of different generations of poly(amidoamine) dendrimers modified with various terminal groups," Analytical Chemistry, vol. 77, no. 7, pp. 2063-2070, 2005.
[25] X. Shi, I. Bányai, K. Rodriguez et al., "Electrophoretic mobility and molecular distribution studies of poly(amidoamine)dendrimers of defined charges," Electrophoresis, vol. 27, no. 9, pp. 1758-1767, 2006.

[26] X. Shi, I. Bányai, M. T. Islam et al., "Generational, skeletal and substitutional diversities in generation one poly(amidoamine) dendrimers," Polymer, vol. 46, no. 9, pp. 3022-3034, 2005.

[27] X. Shi, X. Bi, T. R. Ganser et al., "HPLC analysis of functionalized poly(amidoamine) dendrimers and the interaction between a folate-dendrimer conjugate and folate binding protein," Analyst, vol. 131, no. 7, pp. 842-848, 2006.

[28] N. Sato, H. Kobayashi, T. Saga et al., "Tumor targeting and imaging of intraperitoneal tumors by use of antisense oligoDNA complexed with dendrimers and/or avidin in mice," Clinical Cancer Research, vol. 7, no. 11, pp. 3606-3612, 2001.

[29] D. S. Wilbur, P. M. Pathare, D. K. Hamlin, K. R. Buhler, and R. L. Vessella, "Biotin reagents for antibody pretargeting. 3. Synthesis, radioiodination, and evaluation of biotinylated starburst dendrimers," Bioconjugate Chemistry, vol. 9, no. 6, pp. 813-825, 1998.

[30] H. C. Yoon, M. Y. Hong, and H. S. Kim, "Affinity biosensor for avidin using a double functionalized dendrimer monolayer on a gold electrode," Analytical Biochemistry, vol. 282, no. 1, pp. 121-128, 2000.

[31] R. Spector and D. Mock, "Biotin transport through the bloodbrain barrier," Journal of Neurochemistry, vol. 48, no. 2, pp. 400-404, 1987.

[32] S. Beg, A. Samad, M. I. Alam, and I. Nazish, "Dendrimers as novel systems for delivery of neuropharmaceuticals to the brain," CNS and Neurological Disorders, vol. 10, no. 5, pp. 576$588,2011$.

[33] N. L. Rosi and C. A. Mirkin, "Nanostructures in biodiagnostics," Chemical Reviews, vol. 105, no. 4, pp. 1547-1562, 2005.

[34] B. Basnar and I. Willner, "Dip-pen-nanolithographic patterning of metallic, semiconductor, and metal oxide nanostructures on surfaces," Small, vol. 5, no. 1, pp. 28-44, 2009.

[35] A. N. Shipway, E. Katz, and I. Willner, "Nanoparticle arrays on surfaces for electronic, optical, and sensor applications," ChemPhysChem, vol. 1, no. 1, pp. 18-52, 2000.

[36] C. A. Cason, S. A. Oehrle, T. A. Fabre et al., "Improved methodology for monitoring poly(amidoamine) dendrimers surface transformations and product quality by ultra performance liquid chromatography," Journal of Nanomaterials, vol. 2008, Article ID 456082, 7 pages, 2008.

[37] J. D. Driskell, K. M. Kwarta, R. J. Lipert, M. D. Porter, J. D. Neill, and J. F. Ridpath, "Low-level detection of viral pathogens by a surface-enhanced Raman scattering based immunoassay," Analytical Chemistry, vol. 77, no. 19, pp. 6147-6154, 2005.

[38] J. D. Driskell, J. M. Uhlenkamp, R. J. Lipert, and M. D. Porter, "Surface-enhanced Raman scattering immunoassays using a rotated capture substrate," Analytical Chemistry, vol. 79, no. 11, pp. 4141-4148, 2007.

[39] R. L. Millen, J. Nordling, H. A. Bullen, M. D. Porter, M. Tondra, and M. C. Granger, "Giant magenetoresistive sensors. 2. Detection of biorecognition events at self-referencing and magnetically tagged arrays," Analytical Chemistry, vol. 80, no. 21, pp. 7940-7946, 2008.

[40] D. E. Q. Yang, Y. U. Q. Xiong, Y. Guo, D. A. Da, and W. G. $\mathrm{Lu}$, "Sizes correction on AFM images of nanometer spherical particles," Journal of Materials Science, vol. 36, no. 1, pp. 263267, 2001.

[41] P. Markiewicz and M. C. Goh, "Simulation of atomic force microscope tip-sample/sample-tip reconstruction," Journal of 
Vacuum Science and Technology B, vol. 13, no. 3, pp. 11151118, 1995.

[42] X. C. Wu, A. M. Bittner, and K. Kern, "Microcontact printing of CDS/dendrimer nanocomposite patterns on silicon wafers," Advanced Materials, vol. 16, no. 5, pp. 413-417, 2004.

[43] R. McKendry, W. T. S. Huck, B. Weeks, M. Fiorini, C. Abell, and T. Rayment, "Creating nanoscale patterns of dendrimers on silicon surfaces with dip-pen nanolithography," Nano Letters, vol. 2, no. 7, pp. 713-716, 2002.

[44] P. Bhatnagar, S. S. Mark, I. Kim et al., "Dendrimer-Scaffoldbased electron-beam patterning of biomolecules," Advanced Materials, vol. 18, no. 3, pp. 315-319, 2006. 


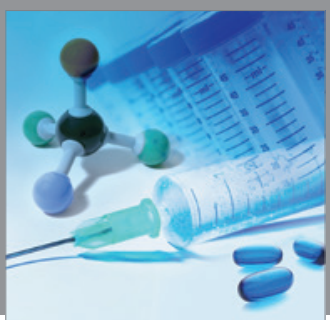

International Journal of

Medicinal Chemistry

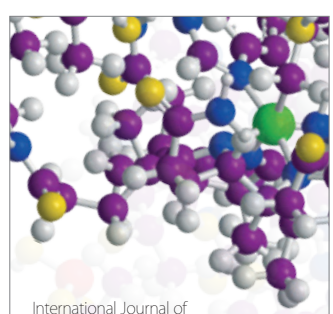

Carbohydrate Chemistry

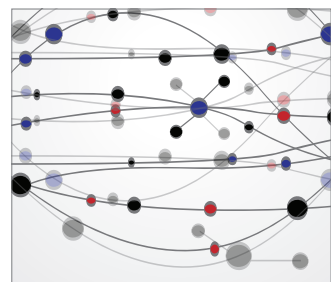

The Scientific World Journal
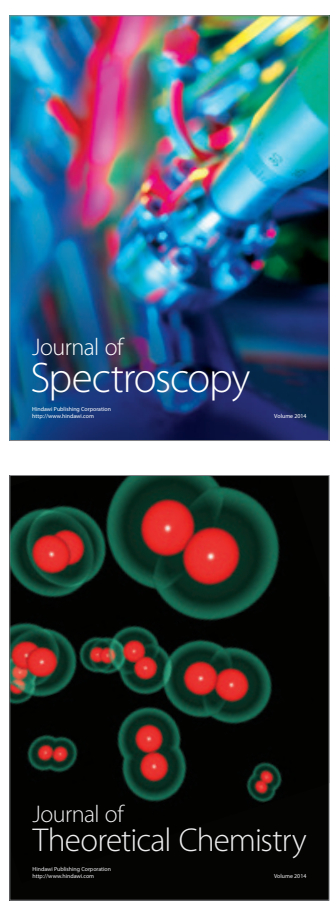
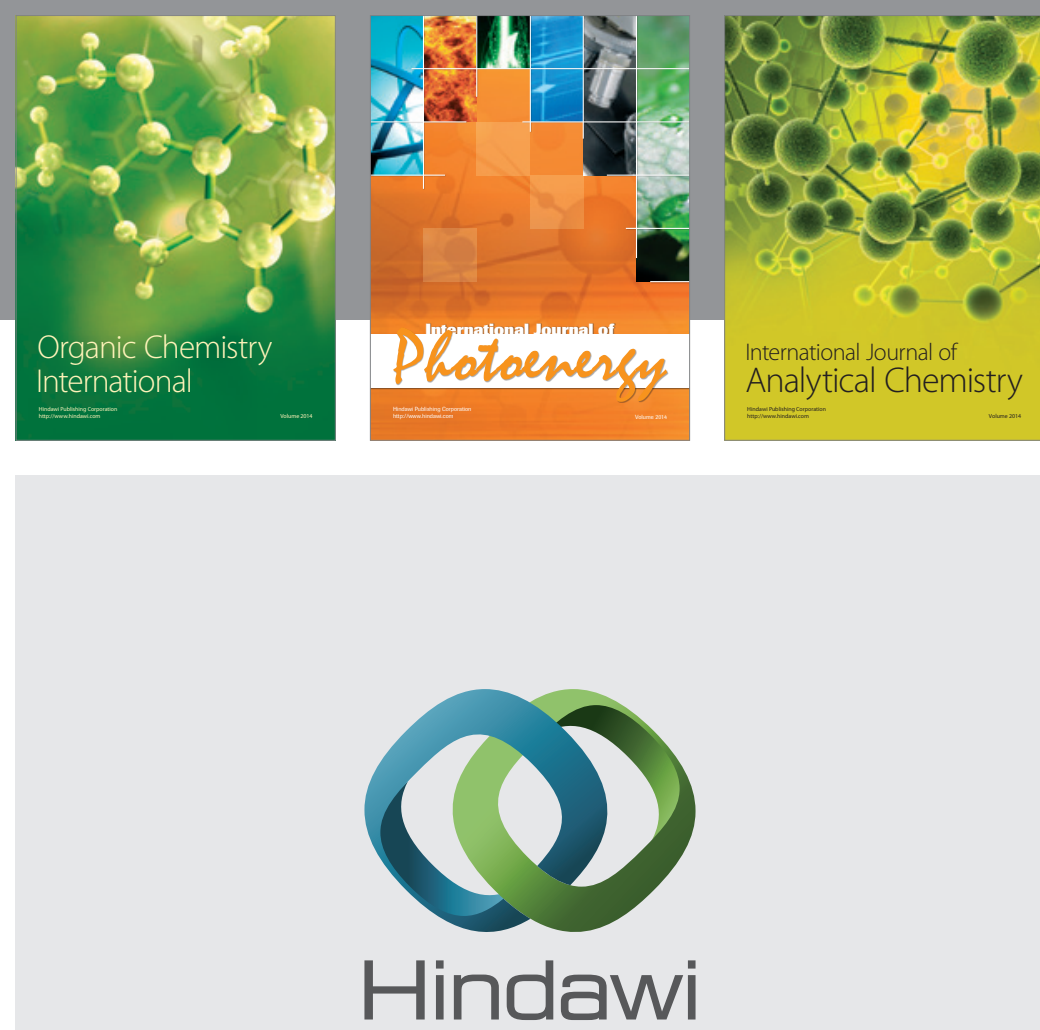

Submit your manuscripts at

http://www.hindawi.com
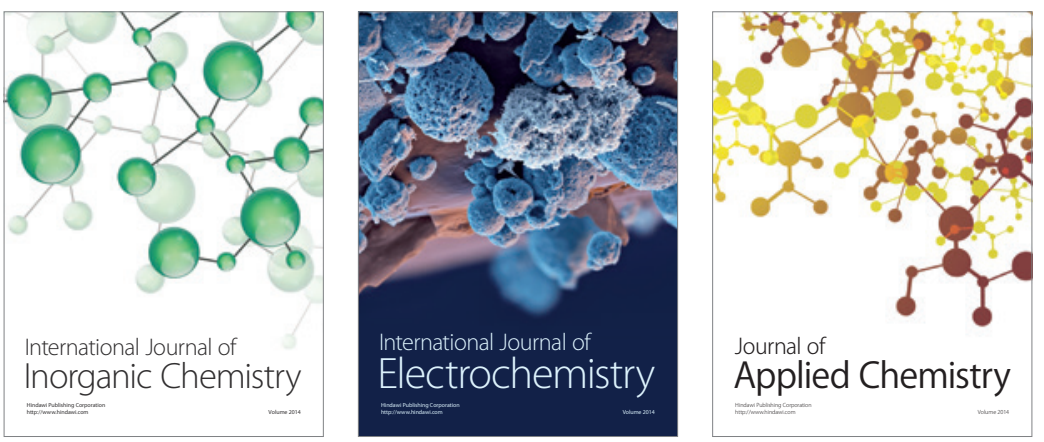

Journal of

Applied Chemistry
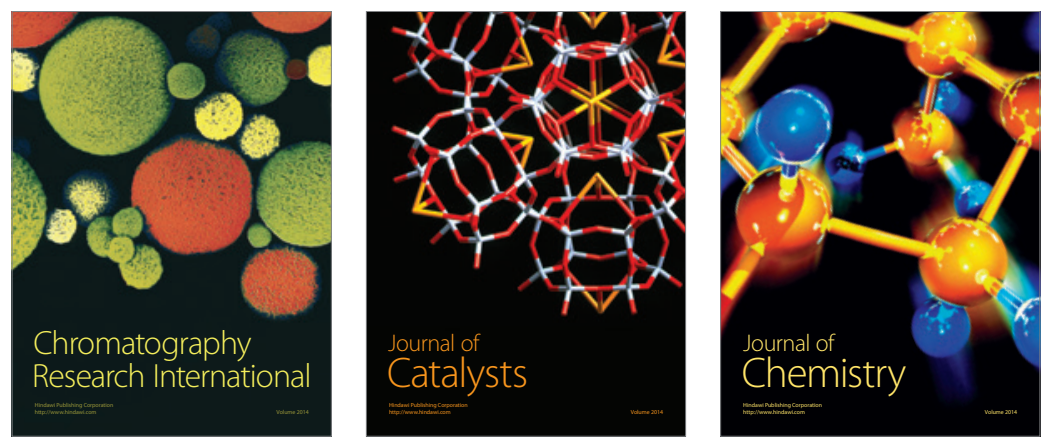
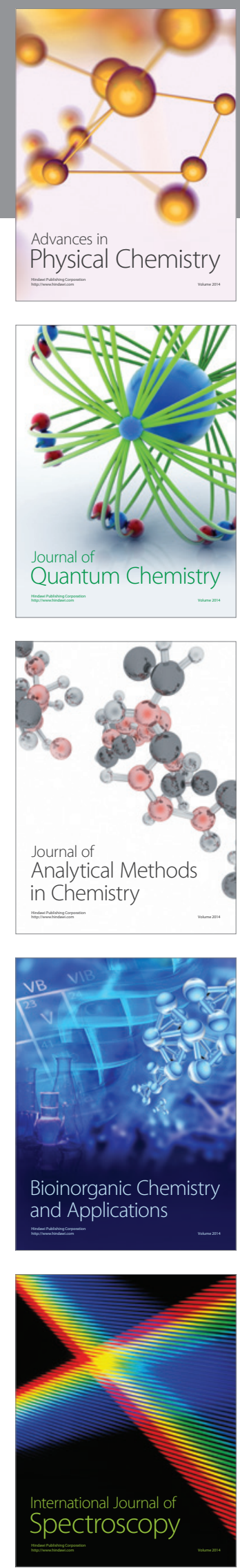